\title{
Scintigraphic detection of sinusitis
}

\section{Lorraine M. Fig and Barry L. Shulkin}

Division of Nuclear Medicine, Department of Internal Medicine, University of Michigan Hospitals, B1G412 Box 0028, Ann Arbor, MI 48109-0028, USA

\begin{abstract}
Radionuclide scintigraphy is useful in the diagnosis of sinusitis. Three cases are described, using different radionuclide agents $\left({ }^{99 m} \mathrm{Tc}-\mathrm{MDP},{ }^{67} \mathrm{Ga}\right.$-citrate and ${ }^{111} \mathrm{In}$ leukocytes) in which sinusitis was discovered, though clinically unsuspected. The advantages and limitations of these agents are briefly reviewed.
\end{abstract}

Key words: Sinusitis - Bone scan - Gallium - Indium leukocyte

Sinusitis is a common clinical problem which may be occult in both adults and children. Incidental detection of sinus infection may occur when scanning is performed with a variety of radionuclide agents. We describe three cases in which radionuclide imaging led to the unsuspected diagnosis of sinusitis.

\section{Material and methods}

Case history 1. An 18-month-old girl with multiple medical problems including congenital asplenia, cardiac anomalies and developmental delay, underwent gallium scanning in search of a source for her unexplained fever and leukocytosis. Increased uptake is seen in the region of the ethmoid sinuses (Fig. 1). Plain radiography was confirmatory for chronic sinusitis.

Case history 2. A 14-year-old boy sustained a gunshot wound to the chin. Bullet fragments lodged in the vertebral body of $\mathrm{C} 4$, rendering him quadriplegic. After laminectomy, intermittent fevers developed, with no obvious source, and a ${ }^{111}$ In labelled autologous leukocyte scan was performed to rule out osteomyelitis in the cervical spine. This revealed increased tracer activity in the right maxillary, frontal and ethmoid sinuses (Fig. 2). Radiographs of these sinuses showed opacity, confirming sinusitis (Fig. 3). Amoxicillin was begun with prompt resolution of fever.

Case history 3. A 69-year-old man underwent thoracotomy for carcinoma of the lung. Two months following lobectomy, a bone scan was requested to evaluate the patient's low back pain. Increased uptake is seen in the region of the ethmoids, right frontal and right maxillary sinuses

Offprint requests to: B.L. Shulkin

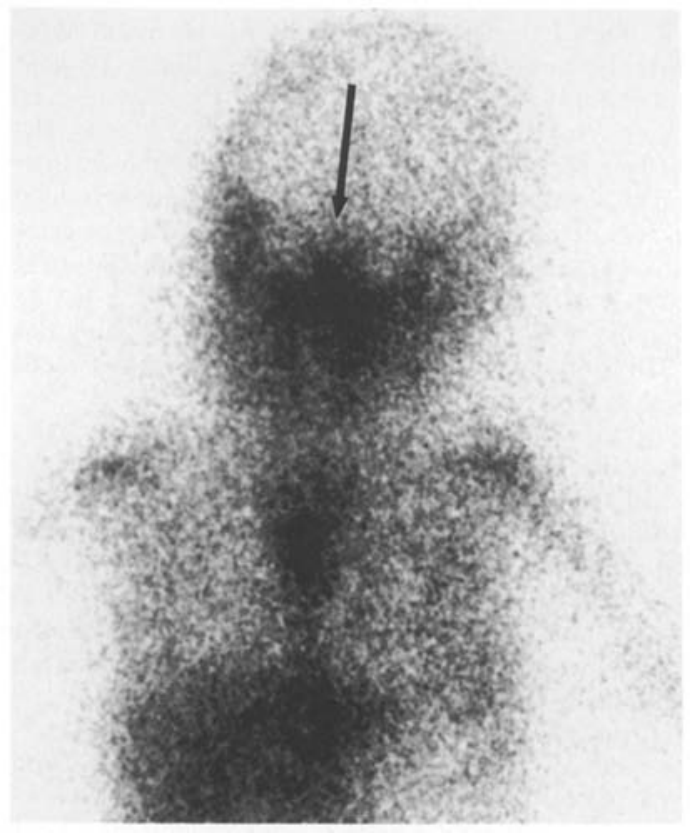

Fig. 1. Anterior image obtained $48 \mathrm{~h}$ following injection of ${ }^{67} \mathrm{Ga}-$ citrate. Increased activity is seen in the region of the right ethmoid sinus (arrow)

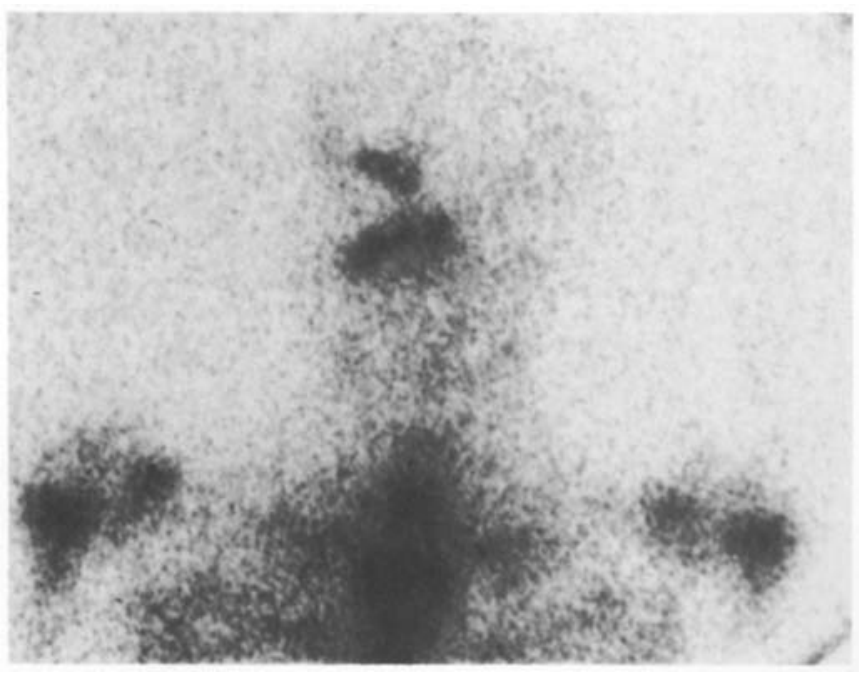

Fig. 2. Anterior image obtained $24 \mathrm{~h}$ following administration of ${ }^{111}$ In-labelled leukocytes. Abnormal tracer accumulation is seen in the right frontal, right maxillary, and ethmoid sinuses 


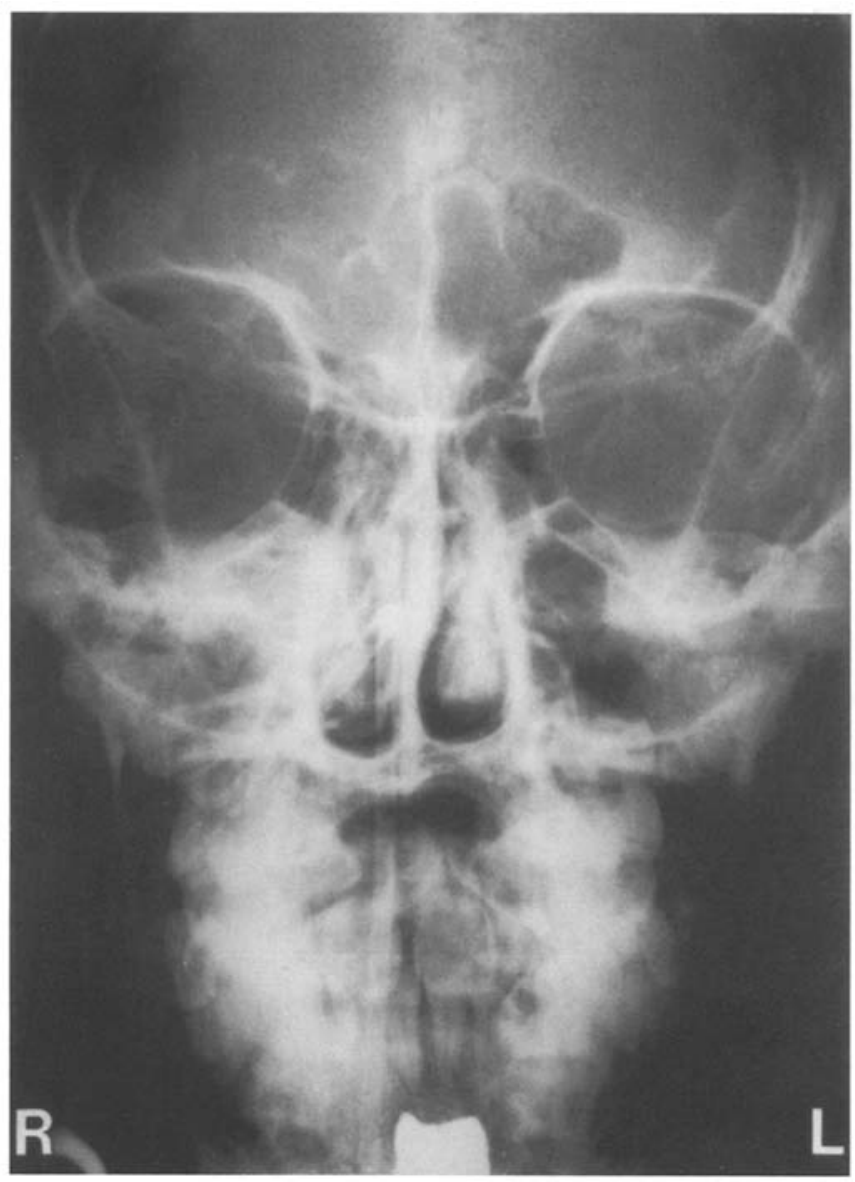

Fig. 3. Radiograph of patient 2 confirms the scintigraphic findings of sinusitis. Opacification of the right frontal, maxillary, and ethmoid sinuses is evident. Sinusitis may be associated with nasogastric tubes

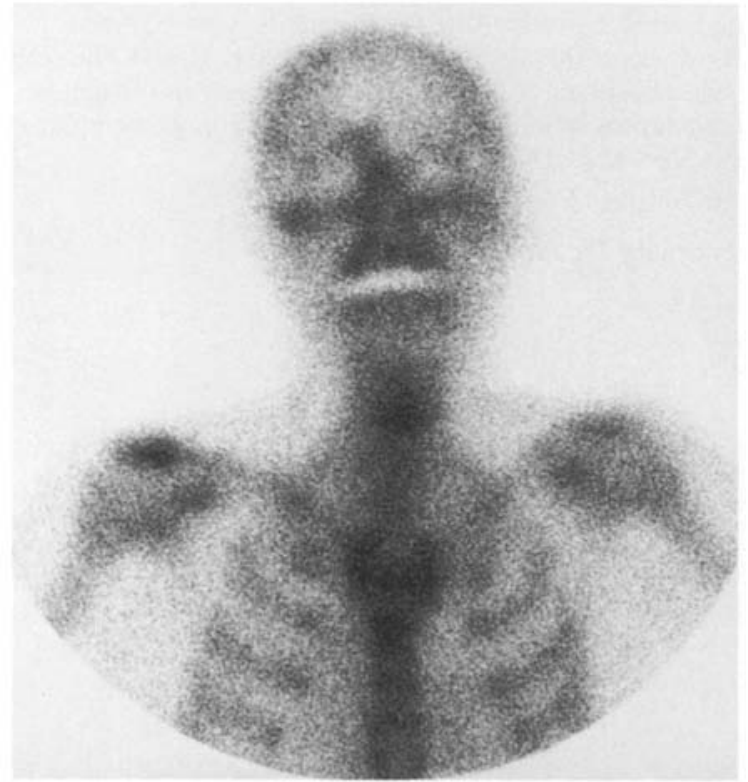

Fig. 4. Anterior image obtained $2 \mathrm{~h}$ after injection of ${ }^{99 \mathrm{~m}} \mathrm{Tc}-\mathrm{MDP}$. Increased tracer uptake is noted in the region of the right maxillary, ethmoid, and frontal sinuses

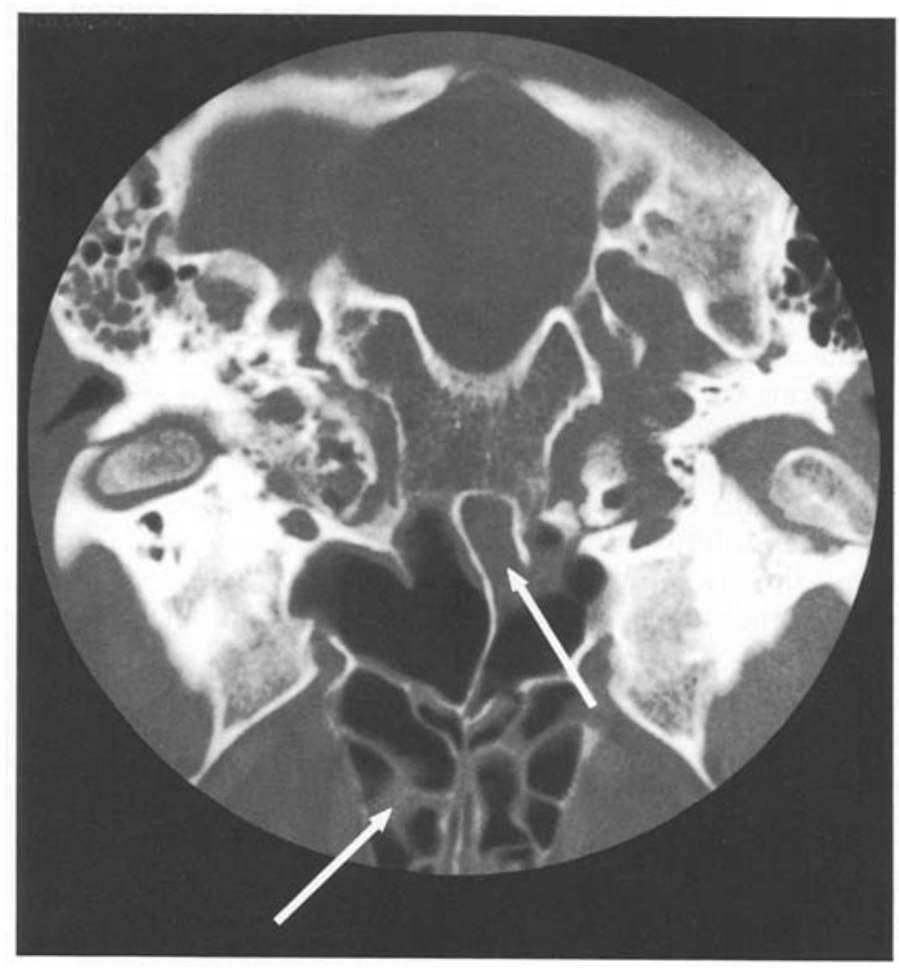

Fig. 5. CT image of patient 3 shows soft tissue thickening in the sphenoid and ethmoid sinuses (arrows), consistent with chronic sinusitis

(Fig. 4). Computed tomography showed soft tissue thickening, confirming the scintigraphic findings of sinusitis (Fig. 5).

\section{Discussion}

Sinusitis is a common problem complicating $0.5 \%$ of upper respiratory infections (Dingle et al. 1964). Acute sinusitis is usually characterized by fever, headache, tenderness to palpation over the affected area and mucopurulent nasal discharge, but at the other end of the spectrum, it may occasionally have a silent presentation (Kern 1984; Friedman and Slavin 1984). The wide range of presenting symptoms and signs may cause delay in making the diagnosis. Untreated, its consequences are potentially severe, with complications such as osteomyelitis, cavernous sinus thrombosis and cerebral abscess occasionally occurring (Harrington 1984). The diagnosis of sinusitis is most often made by the typical clinical history and examination, with or without plain radiography. However, as these cases demonstrate, the diagnosis of sinusitis may also be made incidentally by radionuclide scanning, using gallium, indium labelled leukocytes or bone tracers.

Radiographic studies, while often helpful in diagnosing sinusitis by the appearance of opacity, air fluid levels and mucosal thickening, are generally poor in demonstrating bony involvement (O'Mara 1985) or spread of the infectious process (Ostfeld et al. 1982). Routine sinus radiographs fail to show bony changes for at least seven to ten days in the presence of acute osteomyelitis, and the same problem exists with CT scanning in the absence of bony erosion (Gardiner 1986). On the other hand, facial bone scintigraphy is much more sensitive in determining whether bony 
involvement is present (Bergstedt 1979a). It has been shown that the appearance of increased tracer uptake in bone surrounding infected sinuses is due to a high concentration of the agent in bone tissue, although it is difficult to differentiate true osteomyelitis from bony reaction to purulent sinusitis (O'Mara 1985). Markedly positive scans, especially when tracer uptake occurs beyond the margins of the sinuses, are highly suggestive of acute osteomyelitis (Gardiner 1986). Bone scanning will help in differentiating allergic mucosal swelling from purulent infection, the radiologic appearance of these conditions being identical (Bergstedt $1979 \mathrm{~b}$ )

Gallium scanning in sinusitis is useful in separating osteomyelitis from other disorders, such as trauma, and may also be helpful in assessing the response to antibiotic therapy (Shafer et al. 1981) since absence of uptake in the gallium scan indicates resolution of active infection (Gardiner 1986).

Positive indium leukocyte scanning may occur in the facial region due to sinusitis (Hawker et al. 1985). Other relatively common causes of facial uptake include gingivitis and apical and periodontal abscesses (McAfee and Samin 1985). Active accumulation should be distinguished from non specific blood pool activity; increased midline activity is normal due to the vascularity of the nasal mucosa (Doherty and Goodwin 1981).

Controversy exists concerning the diagnosis of sinusitis in children because it is difficult to assess the significance of sinus opacification as seen on radiographic studies (Dingle et al. 1964; Harrington 1984). While the maxillary and ethmoid sinuses are aerated in infancy, the sphenoid sinus undergoes pneumatization by the third to fifth year of life and the frontal sinuses do not become aerated until the sixth to tenth year (Dingle et al. 1964; O'Mara 1985). Bone scanning may be helpful in making a diagnosis of sinusitis. However, in children, the interpretation of scans may be difficult due to uptake of the tracer in the region of the sinuses due to bony growth, and additional diagnostic procedures may be warranted depending on the clinical situation.

Comparison of the dosimetry among the various radionuclide and radiologic methods available for the diagnosis of sinusitis is difficult since the target organ depends on the radionuclide employed and because the exposure from radiologic procedures is regional. Based on usual adult radiopharmaceutical dosage, the total body absorbed dose in rads are as follows: bone scan -0.26 rads, gallium scan
-0.8 rads, Indium leukocytes -0.25 rads (Carey et al. 1983.). This is compared with plain radiography which imparts 0.22 rads and 0.03 rads respectively to the thyroid gland and active marrow, and head CT which delivers $0.14 \mathrm{rad}$ to the active marrow (Coulam et al. 1981).

In summary, radionuclide scintigraphy is uncommonly utilized in the diagnosis and follow up of treatment of sinusitis. Although each technique has some limitations, they provide valuable information about the sinuses which can lead to the diagnosis of sinusitis and appropriate therapy.

\section{References}

Bergstedt HF, Carenfeldt C, Lind MG (1979a) Facial bone scintigraphy IV. Diagnosis of bone involvement by purulent sinusitis. Acta Radiol Diagn 20:379-384

Bergstedt HF, Carenfeldt C, Lind MG (1979b) Facial bone scintigraphy V. Differentiation of purulent from non-purulent inflammation of the maxillary sinus. Acta Radiol Diagn 20:458-464

Carey JE, Kline RC, Keyes JW (1983) Manual of Nuclear Medicine Procedures, 4th Edition. CRC Press

Coulam CM, Erickson JJ, Rollo FD, James AE (1981) The Physical Basis of Medical Imaging. Appleton-Century-Croft, New York

Dingle JH, Badger GF, Jordan WS Jr (1964) Illness in the home: A study of 25,000 illnesses in a group of Cleveland families. The Press of Case Western Reserve, Cleveland, p 347

Doherty PW, Goodwin DA (1981) Migration of 111-In-labelled granulocytes to infection sites (letter). $\mathrm{N}$ Engl J Med $304: 1546-1547$

Fricdman WH, Slavin RG (1984) Diagnosis and medical and surgical treatment of sinusitis in adults. Clin Rev Allergy 2:409-428

Gardiner LJ (1986) Complicated frontal sinusitis. Evaluation and management. Otolaryngol Head Neck Surg 95:333-343

Harrington PC (1984) Complications of sinusitis. Ear Nose Throat J $63: 58-71$

Kern EB (1984) Sinusitis. J Allergy Clin Immunol 73:25-31

McAfee JG, Samin A (1985) In-111 labelled leukocytes: a review of problems in image interpretation. Radiology 155:221-229

O'Mara RE (1985) Role of bone scanning in dental and maxillofacial disorders. In: Nuclear Medicine Annual. Freeman LM, Weissman HS (eds) Raven Press, New York, pp 265-284

Ostfeld E, Aviel A, Pelet D (1982) Bone scintigraphic diagnosis in acute frontal sinusitis. Acta Otolaryngology 94:557-561

Shafer RB, Marlette JM, Browne GA, Elson MK (1981) The role of Tc $99 \mathrm{~m}$ phosphate complexes and Gallium-67 in the diagnosis and management of maxillofacial disease: concise communication. J Nucl Med 22:8-11

Received February 17, 1988 / May 16, 1988 